\title{
Continuum damage mechanics based approach to the fatigue life prediction of cast aluminium alloy with considering the effect of porosity
}

\author{
Xiaojia Wang ${ }^{1}$, Weiping $\mathrm{Hu}^{2, *}$, and Qingchun $\mathrm{Meng}^{3}$ \\ ${ }^{1}$ Institute of Solid Mechanics, School of Aeronautic Science and Engineering, Beihang University, China \\ ${ }^{2}$ Institute of Solid Mechanics, School of Aeronautic Science and Engineering, Beihang University, China \\ ${ }^{3}$ Institute of Solid Mechanics, School of Aeronautic Science and Engineering, Beihang University, China
}

\begin{abstract}
A damage mechanics based approach is applied for the study of fatigue behaviour of high pressure die cast ADC12 aluminium alloy. A damage coupled elastoplastic constitutive model is presented according to the concept of effective stress and the hypothesis of strain equivalence. An elastic fatigue damage model taking into account the pore-induced stress concentration is developed to investigate fatigue damage evolution of the specimens subjected to cyclic loading. The predicted lives for the specimens with different sizes of pores are consistent with the experimental data. The pore-induced fatigue damage and the variation of fatigue life along with the size of pores are also investigated.
\end{abstract}

\section{Introduction}

In current industrial practice, cast aluminium alloy is widely applied in the automotive and aerospace industries due to their advantage of lightweight, relatively high efficiency, and low cost. However, various kinds of microscopic defects, such as porosities, silicon particles, and cold flakes, are known to be an obstacle concerning fatigue resistance [1]. Among these defects, porosity is one of the most typical forms and inevitably formed during the casting process. Many experimental studies have investigated that the stress concentrations induced by pores become the primary cause of initiation of fatigue cracks and have a considerable influence on fatigue failure [2-3]. Therefore, the effect of porosities is of significant concern in assessing the fatigue life of cast aluminium alloy.

Presently, numerous methods have been adopted to assess the fatigue life of cast aluminium alloy with considering the effect of defects. The most commonly used methods are Murakami's square-root area parameter model [4] and some crack growth models [56]. These models have been applied to predict the fatigue life based on the configuration characters such as the maximum area or diameter of defects. However, there are few damage mechanics models are developed to consider the effect of porosity on the fatigue damage of the cast aluminium alloy. An approach based on continuum damage mechanics (CDM) can not only describe mechanical behaviour of a deteriorated material at the macroscopic scale by introducing damage variables, but also evaluate the progressive damage based on the evolution laws derived from thermodynamics.

This study is aimed at predicting the high cycle fatigue life of specimens obtained from high pressure die-cast ADC12 aluminium alloy by using a continuum damage mechanics based approach with considering the effect of porosity. First, from the framework of CDM, a macroscopic scalar damage variable $D$ is introduced to describe the mechanical deterioration of material under cyclic loading. Second, a fatigue damage model, which includes an elasto-plastic constitutive model and an elastic damage evolution model, is developed to evaluate the fatigue damage of cast aluminium alloy with different sizes of pores under cyclic loading. The damage coupled elasto-plastic constitutive equations are adopted as the material model according to the concept of effective stress and the hypothesis of strain equivalence. Then, a dimensionless quantity related to the diameter of pore is introduced to the damage evolution equations to reflect the effect of stress concentration induced by a pore. It is found that the calculated fatigue lives are in accordance with the experimental results [7].

\section{Fatigue damage model}

\subsection{Damage-coupled elastic-plastic constitutive model}

In the framework of CDM, the deterioration of material can be represented by the damage variable. The damage variable $D$ for the case of isotropic damage of isotropic

Corresponding author: huweiping@buaa.edu.cn 
materials, can be represented by the deterioration ratio of the stiffness of representative volume element (RVE):

$$
D=\frac{E-E_{D}}{E},
$$

where $E$ is the Young's Modulus of the material and $E_{D}$ is the effective Young's Modulus of the RVE with damage. The value of $E_{D}$ ranges from $E$ to 0 , and the value of $D$ varies between 0 and 1 .

For the case of a small deformation, the total strain $\varepsilon_{i j}$ can be decomposed into:

$$
\varepsilon_{i j}=\varepsilon_{i j}^{e}+\varepsilon_{i j}^{p},
$$

where $\varepsilon_{i j}^{e}$ and $\varepsilon_{i j}^{p}$ are the elastic and plastic strains, respectively.

Based on the hypothesis of strain equivalence, the elastic strain of damaged material takes the form of:

$$
\varepsilon^{e}=\frac{1+v}{E}\left(\frac{\sigma_{i j}}{1-D}\right)-\frac{v}{E}\left(\frac{\sigma_{i j} \delta_{i j}}{1-D}\right)
$$

where $E, v$ are the Young's Modulus, Poisson's ratio respectively, The variable $\sigma_{i j}$ is the Cauchy stress of the damaged material. $\delta_{i j}$ is the Kronecker delta: $\delta_{i j}=1, i=j$; $\delta_{i j}=0, i \neq j$ and the subscripts $i$ and $j$ have values of 1,2 , or 3 .

The evolution of plastic strain is defined as:

$$
\dot{\varepsilon}_{i j}^{p}=\dot{\lambda} \frac{\partial f}{\partial \sigma_{i j}},
$$

where $\lambda$ is the plastic multiplier. $f$ is the von Mises yield function with damage and the nonlinear kinematic hardening model proposed by Chaboche [8] is expressed as:

$$
f=\sqrt{\frac{3}{2}\left(\frac{S_{i j}}{1-D}-\alpha_{i j}\right)\left(\frac{S_{i j}}{1-D}-\alpha_{i j}\right)}-Q,
$$

where $S_{i j}$ is the deviatoric part of the stress. $Q$ is the radius of the yield surface. $\alpha_{i j}$ is the deviatoric part of the back stress.

The evolution of plastic strain components [9] can be obtained as:

$$
\begin{gathered}
\dot{\varepsilon}^{p}=\frac{3}{2} \frac{\dot{\lambda}}{1-D} \frac{S_{i j} /(1-D)-\alpha_{i j}}{\left(S_{i j} /(1-D)-\alpha_{i j}\right)_{e q}}, \\
\dot{p}=\sqrt{\frac{2}{3} \dot{\varepsilon}_{i j}^{n} \dot{\varepsilon}_{i j}^{n}}=\left(\frac{\dot{\lambda}}{1-D}\right),
\end{gathered}
$$

the kinematic hardening law is expressed as:

$$
\alpha_{i j}=\sum_{k=1}^{K} \alpha_{i j}^{(k)} \text {, }
$$

$$
\dot{\alpha}_{i j}^{(k)}=(1-D)\left(\frac{2}{3} C_{k} \dot{\varepsilon}_{i j}^{p}-\gamma_{k} \alpha_{i j}^{(k)} \dot{p}\right),
$$

where $K$ is the number of the back stress components. Parameters $C_{k}$ and $\gamma_{k}$ are material constants obtained from experiments.

\subsection{Fatigue damage evolution model}

In the regime of high cycle fatigue, the cyclic stress determines the fatigue lifetime of the material. Without considering the effect of defect contained in the material, for the multiaxial stress state, the elastic damage evolution equation is given by [10]:

$$
\frac{d D}{d N}=\left[1-(1-D)^{\beta+1}\right]^{\alpha} \cdot\left[\frac{A_{I I}}{M_{0}\left(1-3 b_{2} \sigma_{H, m}\right)(1-D)}\right]^{\beta},
$$

where $N$ is the number of cycles until failure. $A_{I I}$ is the amplitude of the octahedral shear stress, which is defined by:

$$
A_{I I}=\frac{1}{2}\left[\frac{3}{2}\left(S_{i j, \max }-S_{i j, \min }\right) \cdot\left(S_{i j, \max }-S_{i j, \min }\right)\right]^{1 / 2},
$$

where $S_{i j \text {, max }}$ and $S_{i j \text {, min }}$ are the maximum and the minimum values of the deviatoric stress tensor $i, j$ components during one loading cycle. The variable $\sigma_{H, m}$ is the mean hydrostatic stress and is defined by:

$$
\sigma_{H, m}=\frac{1}{6}[\max (\operatorname{tr}(\sigma))+\min (\operatorname{tr}(\sigma))]
$$

where, $\operatorname{tr}(\sigma)=\frac{1}{3}\left(\sigma_{1}+\sigma_{2}+\sigma_{3}\right)$.

The parameter $\alpha$ is defined by:

$$
\alpha=1-a\left\langle\frac{A_{I I}-A_{I I}^{*}}{\sigma_{u}-\sigma_{e q, \max }}\right\rangle,
$$

The Sines fatigue limit criterion in this model is formulated by:

$$
A_{I I}^{*}=\sigma_{l 0}\left(1-3 b_{1} \sigma_{H, \text { mean }}\right) .
$$

where $\sigma_{e q, \max }$ is the maximum equivalent stress over a loading cycle. $\sigma_{u}$ is the ultimate tensile stress and $\sigma_{l 0}$ is the fatigue limit at the fully reversed loading condition. Five parameters, $a, \beta, M_{0}, b_{1}$ and $b_{2}$ are determined by using plain fatigue tests of standard specimens.

In the above evolution equation, the term $A_{I I} /(1-D)$ can be regarded as the effective stress amplitude, representing the average effect of damage on the reduction of load-resisting area. However, the stress concentration effect of pores is not considered in this model. It was reported that the fatigue crack initiated 
around the pore with maximum diameter in cast aluminium alloy [11], which indicates that the stress concentration induced by pore relates to the fatigue life of specimen. Accordingly, in our study, a dimensionless quantity related to the maximum pore diameter of porosity is introduced to the above evolution equation to describe the stress concentration effect on fatigue life. This method is much simpler than using the stress field obtained by building a meso-scale model of RVE involving a pore. Moreover, considering that the stress concentration factor increases as the pore diameter increases, correspondingly, the fatigue life decreases [12], the damage evolution model for cast aluminium alloy is proposed as:

$$
\frac{d D}{d N}=\left[1-(1-D)^{\beta+1}\right]^{\alpha} \cdot\left[\frac{A_{I I}\left[1+\left(\frac{d_{\max }}{l}\right)^{c}\right]}{M_{0}\left(1-3 b_{2} \sigma_{H, m}\right)(1-D)}\right]^{\beta},
$$

where $d_{\max }$ is the diameter of the largest pore, which can be obtained through 3D X-ray tomography inspection. It should be noted that all the pores are assumed to be spheric for the simplicity. The term $\left[1+\left(\frac{d_{\max }}{l}\right)^{c}\right]$ is used to reflect the effect of stress concentration induced by porosities. $l$ is a characteristic dimension for describing the effect of a pore on the stress field around the pore. The length of $l$ is taken to be about twice as long as the diameter of the largest pore. $c$ is a material parameter that can be determined through the fatigue testing of materials.

\section{Fatigue life prediction of material}

A recent experimental study on die cast aluminium alloy indicated that porosities have a significantly effect on fatigue behaviour. The specimens for the X-ray tomography scan and fatigue test were machined from a practical high pressure die casting (HPDC) ADC12 aluminium alloy [7]. During the die casting process, the melt pouring temperature was $680^{\circ} \mathrm{C}$, and the shot speeds were 0.1 to 0.8 and $4.0 \mathrm{~m} / \mathrm{s}$ during the slow and high injection periods, respectively. The intensification pressure was set to $65 \mathrm{MPa}$. The compositions of the material are presented in Table 1. The fatigue specimens were in accordance with the ASTM standard. The geometry of specimens is shown in Fig. 1. The thickness of specimens is $4 \mathrm{~mm}$. The pores in the gauge segment of all specimens were detected with high resolution X-ray tomography nondestructively before the fatigue test.
Table 1. Chemical composition of the material (wt. \%).

\begin{tabular}{ccccccc}
\hline $\mathrm{Si}$ & $\mathrm{Cu}$ & $\mathrm{Fe}$ & $\mathrm{Zn}$ & $\mathrm{Mn}$ & $\mathrm{Mg}$ & $\mathrm{Al}$ \\
10.77 & 1.87 & 0.78 & 0.78 & 0.2 & 0.17 & Bal. \\
\hline
\end{tabular}

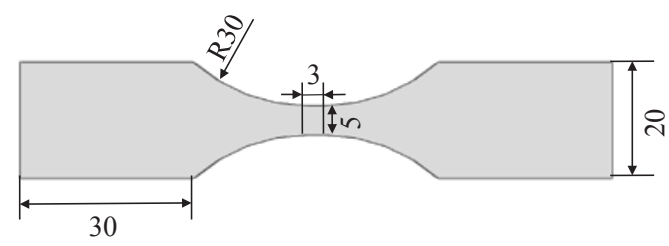

Fig.1. The geometry of the fatigue specimen (all dimensions are in $\mathrm{mm}$ ).

The specimens were classified according to the average porosity of them. The high cycle fatigue tests were carried out at five stress levels of 110, 130, 145, 160 , and $190 \mathrm{MPa}$ for each group. The stress ratio of fatigue loading was $\mathrm{R}=0.1$ and the test frequency was $\mathrm{f}=30 \mathrm{~Hz}$ under the controlled temperature of $20^{\circ} \mathrm{C}$. The fractured surfaces of all specimens were observed with a scanning electron microscope (SEM). Part of the results [7] are shown in Table 2.

Table 2. Experimental results under different pore conditions.

\begin{tabular}{cccc} 
Maximum & Volume & Maximum & fatigue \\
Stress & fractions & diameter(mm) & $\begin{array}{c}\text { life(cycles) } \\
190\end{array}$ \\
$0.63 \%$ & 0.452 & 21856 \\
190 & $0.41 \%$ & 0.301 & 36594 \\
190 & $0.17 \%$ & 0.137 & 134966 \\
160 & $1.02 \%$ & 0.315 & 84886 \\
160 & $0.57 \%$ & 0.171 & 120615 \\
160 & $0.17 \%$ & 0.105 & 260788 \\
145 & $0.65 \%$ & 0.482 & 182796 \\
145 & $0.25 \%$ & 0.136 & 1044904 \\
145 & $0.15 \%$ & 0.201 & 807453 \\
130 & $0.94 \%$ & 0.448 & 372192 \\
130 & $0.45 \%$ & 0.131 & 387274 \\
130 & $0.15 \%$ & - & $>10^{7}$ \\
110 & $0.91 \%$ & 0.367 & 2360642 \\
110 & $0.56 \%$ & - & $>10^{7}$ \\
110 & $0.15 \%$ & - & $>10^{7}$ \\
\hline
\end{tabular}




\subsection{Determination of material parameters for damage analysis}

The stress-strain curve obtained from the uniaxial tensile test [13] shown in Fig. 2 is used to determine the parameters in the elasto-plastic constitutive equations. The integration of Eq. (9) under the uniaxial loading leads to the stress-strain curve expressed as:

$$
\sigma=\sum_{k=1}^{K} \frac{C_{k}}{\gamma_{k}}\left(1-e^{-\gamma_{k} \varepsilon_{p}}\right)+\sigma_{y} \text {. }
$$

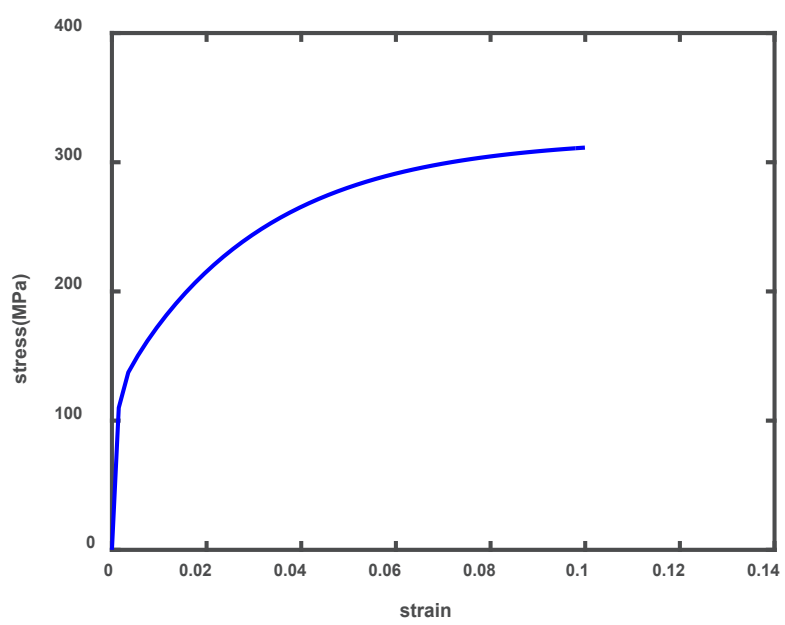

Fig.2. Stress-strain curve for ADC12 cast aluminium alloy.

In this study, three components of back stress are adopted to represent the nonlinear kinematic hardening behaviour. The static mechanical and calculated material parameters of the constitutive model are presented in Table 3.

Table 3. Static mechanical and material parameters for ADC12 cast aluminium alloy.

\begin{tabular}{ccc}
\hline $\mathrm{E}(\mathrm{GPa})$ & $v$ & $\sigma_{y}(\mathrm{MPa})$ \\
76 & 0.3 & 110 \\
$C_{1}(\mathrm{MPa})$ & $C_{2}(\mathrm{MPa})$ & $C_{3}(\mathrm{MPa})$ \\
85610.7 & 2017.5 & 6342.5 \\
$\gamma_{1}$ & $\gamma_{2}$ & $\gamma_{3}$ \\
7053.87 & 373.2 & 33.2 \\
\hline
\end{tabular}

For the elastic damage evolution model, there are five parameters $a, \beta, M_{0}, b_{1}$ and $b_{2}$ needed to be determined. The parameters are determined through fatigue testing of the smooth specimens under uniaxial fatigue loading with different stress ratios. The fatigue test data for ADC12 cast aluminium alloy is taken from the literature [14]. The calculated material parameters in the fatigue damage model are listed in Table 4.
Table 4. Material parameters in the fatigue damage model.

\begin{tabular}{cccccc}
\hline$a$ & $\beta$ & $M_{0}$ & $b_{1}$ & $b_{2}$ & $c$ \\
0.6 & 7.09 & 1129.3 & 0.0176 & 0.00075 & 0.5757
\end{tabular}

The integration of Eq. (15) gives:

$N_{F}=\frac{1}{1+\beta} \cdot \frac{1}{a M_{0}^{-\beta}} \cdot\left\langle\frac{\sigma_{u}-\sigma_{e q, \max }}{A_{I I}-A_{I I}^{*}}\right\rangle\left(\frac{A_{I I}\left[1+\left(\frac{d_{\max }}{l}\right)^{c}\right]}{1-b_{2} \sigma_{H, m}}\right)^{-\beta}$.

The parameter $c$ is needed to determine through the experimental data of fatigue specimens. In this study, the testing data with fatigue life in terms of loading cycles under different pore conditions in Table 3 are used to determine this parameter. The calculated material parameter $c$ is listed in Table 4 . The value of $l$ is taken as $1 \mathrm{~mm}$, which is about twice as long as the diameter of the largest pore, $0.482 \mathrm{~mm}$.

\subsection{Calculation of stress and algorithm of fatigue damage calculation}

In the ABAQUS platform, only $1 / 2$ of the specimen is built with the symmetry boundary conditions at the plane of symmetry. A 3-D 8-node solid element is employed to accomplish the calculation. The FE model after meshing is shown in Fig. 3. A mesh discretization of high quality in the middle region of specimen was performed to accurately acquire the stress field under cyclic loadings. A minimum element size of $0.3 \mathrm{~mm} \times 0.5 \mathrm{~mm} \times 1.0 \mathrm{~mm}$, was adopted to compromise between the accuracy of numerical simulation and the computational time.

The entire numerical computation of the fatigue life of ADC12 cast aluminium alloy includes the calculation of stresses and strains and the elastic-plastic fatigue damage. The flowchart of calculation is showed in Fig. 4.The user-defined subroutines called at all material integration points at the beginning of each time increment are used to implement the damage-coupled elasto-plastic constitutive model and damage evolution model. The detailed computational procedures can be referred in literature [15].

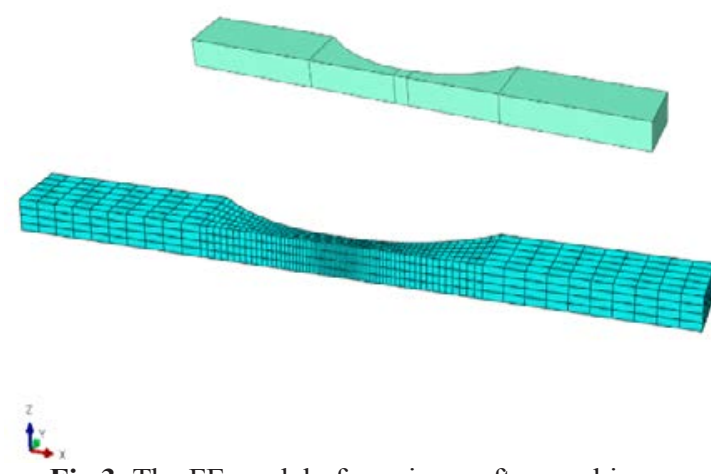

Fig.3. The FE model of specimen after meshing. 


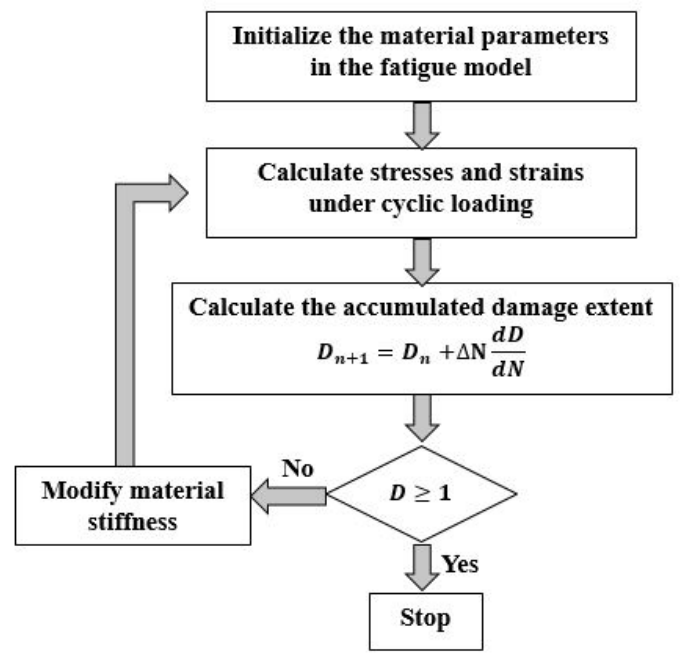

Fig.4. The flowchart for the FE computational methodology.

\section{Results and discussion}

\section{1 fatigue damage analysis}

Fig.5 depicts the damage distribution zone of the specimen right before the initiation of fatigue crack under the conditions $\sigma_{\mathrm{a}}=190 \mathrm{MPa}$ and $\mathrm{d}_{\max }=0.301$. The maximum fatigue damage occurs at the middle of the cross section of specimen.

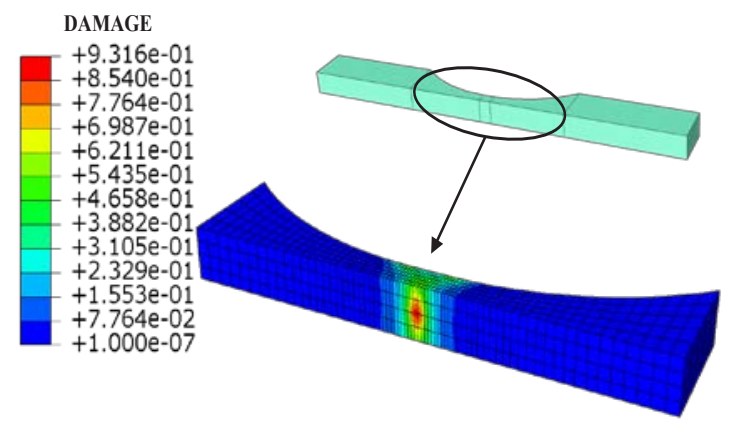

Fig.5. Damage contour on the middle cross section for $\sigma_{\mathrm{a}}=190$ $\mathrm{MPa}, \mathrm{d}_{\max }=0.301$.

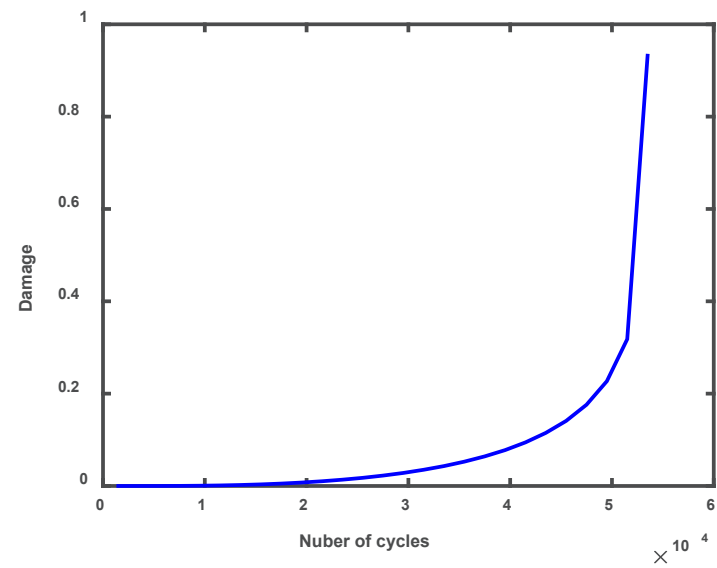

Fig.6. Evolution of the fatigue damage at the critical point for $\sigma_{\mathrm{a}}=190 \mathrm{MPa}, \mathrm{d}_{\max }=0.301$
The extent of damage versus the number of cycles at the critical point is shown in Fig. 6. During a long period of the entire lifetime, the elastic damage increases slowly as the number of cycles increases. After the fatigue damage reaches around 0.2 , it increases significantly, the specimen becomes failure after experiencing a small amount of cycles.

\subsection{Fatigue life results}

Based on the aforementioned computational method, the fatigue lives of specimens with porosities are calculated. Fig.7 illustrates the predicted fatigue lives under different pore conditions and their comparation with the experiment data. It can be seen that the calculated results agree well with the experimental data and all of the data fall within the factor of twice error bands.

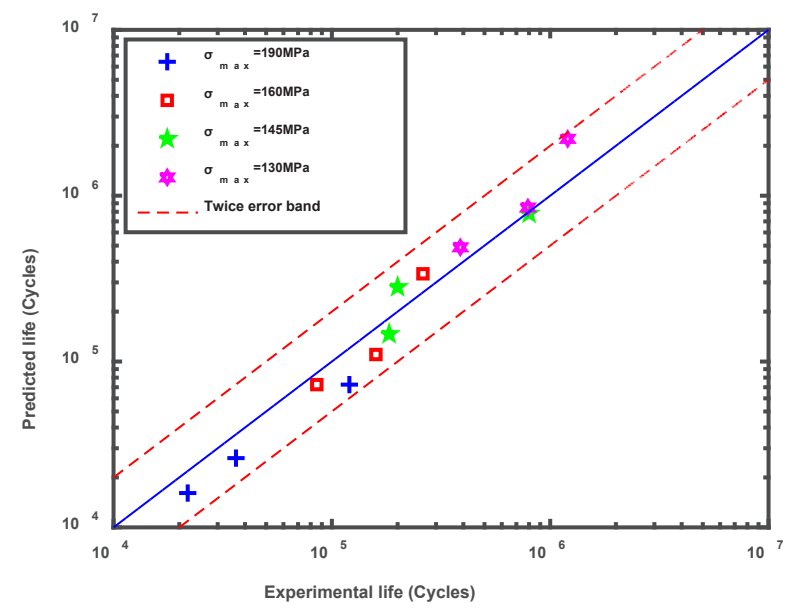

Fig.7. Comparison between the calculated results and experimental data

This fatigue model is then applied to predict the S-N curves of material with different pore conditions. Table 5 illustrates the assumed diameter of porosities of ADC12 cast aluminium alloy and the calculated stress concentration factors induced by pores. Fig. 8 depicts the predicted S-N curves under these conditions. The vertical axis is the applied maximum fatigue stress and the horizontal axis is the logarithm scale of number of failure cycles. It is shown that the fatigue strength decreases as the diameter of the largest pore increases.

Table 5. Stress concentration factor under varies pore conditions.

$\begin{array}{llll}d_{\max }(\mathrm{mm}) & 0.1 & 0.3 & 0.5 \\ 1+\left(\frac{d_{\max }}{l}\right)^{c} & 1.267 & 1.500 & 1.671\end{array}$




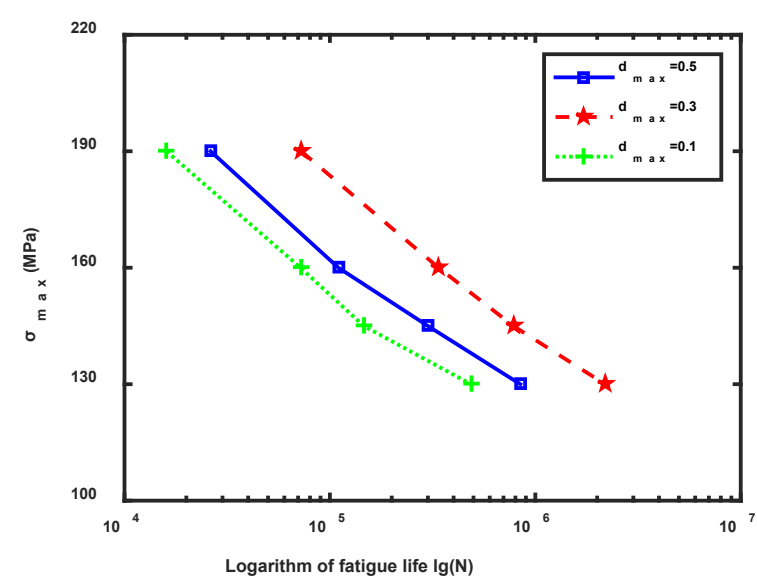

Fig.8. Predicted S-N curves with various pore conditions.

\section{Conclutions}

In this study, a new approach is proposed to calculate the fatigue life of ADC12 cast aluminium alloy considering pore-induced stress concentration based on the continuum damage mechanics theory. The following conclusions can be made:

(1) A dimensionless quantity related to the diameter of the largest pore is employed to the elastic fatigue damage evolution model. The proposed model can be used to well predict the fatigue life of $\mathrm{ADC} 12$ cast aluminum alloy considering the effect of porosity.

(2) During a long period of the entire lifetime, the elastic damage increases slowly as the number of cycles increases. After the fatigue damage reaches around 0.2 , it increases significantly, the specimen becomes failure after experiencing a small amount of cycles.

(3) The calculated fatigue lives of ADC12 cast aluminum alloy under different sizes of pores are in accordance with the experimental data available in the literature. Moreover, the predicted S-N curves provide an important reference to investigate the fatigue property of ADC12 cast aluminum alloy.

\section{References}

1. Y.X. Gao, et al., Acta Mater, 52(19):5435-5449 (2004).

2. Q. G. Wang, D. Apelian and D. A. Lados, J. Light Met,1:73-84(2001).

3. P.Wu,Y.Nadot, et al. Int. J Fatigue,63:97109(2014).

4. Y. Murakami, M. Endo, Int. J Fatigue,16(1994).

5. J. P. Weiler, J. T. Wood, R. J. Klassen, E. Maire, R. Berkmortel and G. Wang, Mater. Sci. Eng. A, 395, 315(2005).

6. H. Hu, M. Zhou, Z. Sun and N. Li. J. Mater. Process. Technol., 201, 364(2008).
7. Q. Wan, H.D. Zhao and C. Zou, ISIJ Int., 54,511515 (2014).

8. J. L. Chaboche, Int. J Plasticity, 7: 661-678 (1991).

9. J. Lemaitre, J. L. Chaboche, Mechanics of solid materials. (Cambridge University Press, 1990).

10. J. L. Chaboche, P.M. Lesne, Fatigue Fract Eng Mater Struct,11(1):1-17(1988).

11. K.S. Chan, Int. J Fatigue. 32(9):1428-1447(2010).

12. F. Bouafia, S. Boualem, MM. El Amin, B. Benali. Comput Mater Sci,50(4):1450-1459(2011).

13. C. Dørum, H. I. Laukli, O. S. Hopperstad, M. Langseth, Eur J Mech A-Solid, 28:1-13(2009).

14. O. Kuwazuru, Y. Murata, Y. Hangai, et al., J. Solid Mech.\& Mater. Eng., 2(9):1220-1231(2008).

15. Z.X. Zhan, W.P. Hu, M. Zhang, et al., Int. J Fatigue, 74:173-182(2015). 